\title{
THE ELECTRON BUBBLE IN LIQUID HELIUM
}

\section{P. H. Roberts}

University of Newcastle upon Tyne,

Newcastle upon Tyne, NE1 7RU, England

\section{ABSTRACT}

The bose condensate model of hellum is used to examine the structure of the electron bubble in helium. The solution obtained makes use of the fact that the parameter $(\mathrm{am} / \mathrm{hM})^{1 / 5}$ is small and $\mathrm{m} / \mathrm{M}$ ls negligible, where $\mathrm{m}$ is the electron mass, $M$ is the boson mass, i is the electron-boson scattering length, and $a$ is the healing length. It is shows that, to leading order, the radius of the bubble is $\mathrm{b}=\left(\pi \mathrm{M}^{2} \mathrm{a}^{2} / \mathrm{mo}_{\infty}\right)^{1 / 5}$, when $\rho_{\infty}$ is the belium density. The effects of (quantum) surface tension and of polarization are discussed, and are shown to be small. Consideration is given to the effective mass and radius of the bubble, and the ellipticity induced in it by slow motion is given. The normal modes of pulsation of the bubble are found and the mobility of the ion is computed. The theory is compared with experiment.

\section{TNTRODUCTION}

It has become increasingly apparent over the past decade that the del1berately introduced impurity can be a fruitful experimental probe of the structure and dynamics of helium II, the superfluid phase of hellum. of particular interest is the negative lon which consists of an electron that, through its zero point motion, cagres out a soft bubble of about $16 \AA$ in radius in the surrounding hellum ( $1 A$ $=10^{-8} \mathrm{~cm}$.). The induced hydrodynamic mass of such a large structure is greatly in excess of 1 ts physlcal mass, and it therefore responds to applied forces as would a much more massive fon. The experimental situation has been reviewed by Donnelly', and more recently by Fetter ${ }^{2}$.

The negative lon provides an Interesting and, as we shall see, a sensitive testing ground for theories of helfum II. We examine in this paper one particularly simple model of hellum near absolute zero, the bose condensate. The approach is expounded by, for example, Gros8 ${ }^{3}$ and by Fetter and Walecka. The theory 18 so simple to apply that most of the properties-of the electron bubble can be calculated in an elementary way. We will present our arguments in a hydrodynamic framework originally proposed by Madelung ${ }^{5}$. Since this may be unfamiliar to the reader, it is developed in $\$ 2$ for the simple single-particle Schrodinger equation. It is generalized in $\$ 3$ to the bose condensate. The two theories are brought together in 34, where the theory of the electron bubble is developed. The final section (55) confronts the theory with experiment.

\section{MADELUNG'S TRANSFORMATION}

It appears to have been Madelung 5 who first realized that Schrbdinger's equation could be cast into a fluid mechanical mold, by expressing the wayefunction, $\psi(\vec{x}, t)$, In terms of 1 ts amplitude, $f(\vec{x}, t)$, and phase, $\phi(\vec{x}, t)$. Consider a particie of mass in in a fleld of fixed potential, $w(\dot{x})$, and therefore obeytng

$$
1 \mathrm{H} \partial \psi / \partial t=-\left(\mathrm{I}^{2} / 2 \mathrm{~m}\right) \nabla^{2} \psi t w \psi \text {. }
$$

By writting

$$
\psi=f \exp (1 m \phi / n),
$$


where $f$ and $\phi$ are real, we can divide (1) Into

$$
\begin{aligned}
& 2 \frac{\partial f}{\partial t}+f \nabla^{2} \phi+2 \vec{\nabla} f \cdot \vec{\nabla} \phi=0, \\
& \frac{\partial \phi}{\partial t}+\frac{1}{2}\left(\vec{\nabla}_{\phi}\right)^{2}+\frac{i}{m}-\frac{\pi^{2}}{2 m^{2}} \frac{\nabla^{2} f}{f}=0 .
\end{aligned}
$$

When we introduce the (probabilistic) mass density, $p$, and current $\vec{j}$, by writing

\[ \rho=m|\psi|^{2}=m f^{2}, \vec{j}=(\mathrm{m} / 21)(\psi \]
and define a velocity, $\vec{u}$, by their ratio

$$
\overrightarrow{\mathbf{u}}=\vec{j} / 0=\vec{\nabla} \phi \text {, }
$$

we recognize that (3) and (4) are the continuity and momentum equations governing the potential flow $(6)$ :

$$
\begin{aligned}
& \frac{\partial \rho}{\partial t}+\vec{\nabla} \cdot(\rho \vec{u})=0, \\
& \frac{\partial \phi}{\partial t}+\frac{1}{2} \vec{u}^{2}+\frac{w}{m}+\mu=0,
\end{aligned}
$$

where

$$
\mu=-\left(\pi^{2} / 2 m^{2}\right) \nabla^{2} p^{1 / 2} / p^{1 / 2} \text {. }
$$

There are three main differences from classical potential flow. First, the total quantity of 'fluid' Is not only conserved by (7): it is fixed by

$$
\int|\psi|^{2} d \vec{x}=1 \text {, or } \int \rho d \vec{x}=\text { m. }
$$

Second, even if through the presence of walls $(w-\infty)$ or otherwise the fluid is confined to a certain multiply-connected domain, $R$, $\psi$ must remain singlevalued. It follows from (2) that, round any contour $\Gamma$ in $\beta$ not reducible to a point by a continuou deformation, $\phi$ can change by a multiple of $\mathrm{h} / \mathrm{m}$ only. The circulation round $\Gamma$ cannot freely take any value: 1 is quantized by the Bohr-Sommerfeld condition

$$
\omega_{\Gamma} \vec{u} \cdot d \vec{x}=\frac{n h}{m}, \quad(n=0, \pm 1, \pm 2 \ldots \ldots) \text {. }
$$

Third, a completely new term has appeared in the momentum equation (8), namely $\mu$.

The term $\mu$ is often called 'the quantum pressure'. This is a misnomer for at least three reasons. First, its dimensionality is incorrect, and it would be better regarded as a chemical potential per unit mass. Second, since where ${ }^{6-8}$

$$
\partial \mu / \partial x_{1}=-p^{-1} \partial \sigma_{1 j} / \partial x_{j},
$$

$$
\sigma_{1 j}=\frac{\pi^{2}}{2 m^{2}} \rho \frac{\partial^{2}}{\partial x_{1} \partial x_{j}}\left(\ln \rho^{1 / 2}\right) \text {, }
$$

a rival, and properly dimensioned, contender for the title of quantum pressure exists as part of the unusual and complicated stress tensor (13). Third, the word 'pressure' suggests a phenomenon that depends only on 'the local thermodynamic state' (here fixed by $p$ ), and the presence of derivatives in (9), or (13), shows that all neighboring points are involved in its definition. Despite these objections, we follow the common usage.

As may be seen by setting $\pi=0$ in ( $(9)$, the quantum pressure is the essential ingredient that distinguishes our subject from the classical theory. The fluid dynamlist can gather experience of its effects 
by translating some of the elementary situations of quantum theory lnto thetr corresponding fluld mechantical statements.

'Hydrostat1cs' arlses from the quantum mechanlcal bound states by writing

$$
\phi=-\mathrm{Et} \text {, }
$$

where $E_{2}$ the energy of the state, is a constant. By (8) the quantum pressure balances $\mathrm{E}-\mathrm{w}$ everywhere. It is best to avold the usual flutd mechantcal practice of absorbing $E$ into $w$, since some energy levels may be inaccessible. For example, when (8) is written as

$$
-\left(x^{2} / 2 m\right) \nabla^{2} f=[E-\nabla(\vec{x})] f \text {, }
$$

and it is supposed that $w$ Increases Indefinttely with distance, $r$, from some origin, 0 , one famtly of solutions to (15) is found that increase with $r$, so that the normalization Integrals (10) do not converge. The condition that only the normalizable solutions of the other family are used transforms (15) Into an eigenvalue problem that confines $\mathrm{E}$ to discrete levels. of course a continuum of elgenvalues exists when $w$ is bounded above.

A well-known application of (15), that is particularly relevant to the bubble, is the potential well for whtch

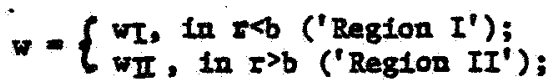

where w and $w_{I}\left(w_{I}\right)$ are constants. Writing

$$
\lambda I^{2}=\left(2 m / \pi^{2}\right)(B-m I), \quad \lambda_{I^{2}}{ }^{2}=\left(2 m / \pi^{2}\right)\left(w_{I}-E\right),
$$

we see that, for $w_{I}<\mathrm{E}<w_{\pi}$, (15) Is obeyed by

$$
\begin{aligned}
& f=f_{I} \equiv A j_{\ell}\left(\lambda_{I} r\right) Y_{\ell}\left(\theta_{,} x\right) \text {, in } r \leq b ; \\
& f=f_{I I} \equiv A k_{\ell}\left(\lambda_{I I} r\right)\left[j_{\ell}\left(\lambda_{I} b\right) / k_{\ell}\left(\lambda_{I I} b\right)\right] Y_{\ell}(\theta, x), \text { in } r \geq b ;
\end{aligned}
$$

where $j_{\ell}(z)$ is the spherical Bessel function of the first kind, $k_{\ell}(z)$ is the modified spherical Bessel function of the second kind, and $Y_{l}(\theta, X)$ is a surface harmonic of integral degree, $l$, in spherical coordinates $(x, \theta, X)$. The exclusion of the other spherical Bessel functions ensures that (10) can be met for some cholce of the constant A. Continulty of $f$ has been realized, and $\nabla_{f}$ is continous provided

$$
\lambda_{I} b j_{l}{ }^{-}\left(\lambda_{I} b\right) / J_{\ell}\left(\lambda_{I} b\right)=\lambda_{I I} b k_{\ell}{ }^{-}\left(\lambda_{I} b\right) / k_{\ell}\left(\lambda_{I I} b\right)
$$

Th1s dispersion relationship determines a discrete spectrum of admissible $\mathrm{E}$. It may be seen that, when $\Delta w \equiv w_{I}$ - wI is large compared wth $\mathrm{h}^{2} / \mathrm{mb}^{2}$, elgensolutions exist for which $E^{-}$.I $<$wII - E. For these, (19) takes the approximate form

$$
f_{I I}=A \exp \left[-\lambda_{I I}(r-b)\right] Y_{L}(\theta ; x), \quad \text { in } r \geq b \text {. }
$$

The flutd is confined in region II to a boundary layer of thickness $1 / \lambda_{\text {II }}$, or

$$
a_{m}=n(2 \pi \Delta \omega)-1 / 2
$$


This phenomenon is often called 'healing', the layer itself a 'healing layer', and $a_{m}$ the 'healing length'. To the fluid mechanicist, the abrupt increase $\Delta \mathrm{w}$ In $\mathrm{at} x=\mathrm{b}$ can only be hydrostatically balanced by an equally abrupt Increase in quantum pressure. It is the non-1ocal character of quantum pressure that causes the fluld to pass through the barrier at $\mathrm{r}-\mathrm{b}$ and, when lower potentials are available externally, permits it to seep out of region I.

In view of later developments, It is worth elaborating the situation just described. First we allow the well to have any shape, definting $n$ to be a coordinate that measures distance from the discontinuity normally outwards from I to II. Second, we allow wI and wII to vary, and suppose that the large transition from wI up to WII does not occur abrupely as in (16), but continuously over some distance, $a$, comparable with $a_{m}$. Within this distance, there is evidentiy no unique way of defining 'the' surface, $S$, of the well. We note, however, that in the case of discontinuous w just considered, $j_{\ell}\left(\lambda_{I} b\right)$ is small, by $(20)$. This suggests we should locate $S$ on the surface of zero $f_{I}$.

To elucidate the healing layer structure, we introduce a stretched coordinate, $\xi$, and cast (15) into dimensionless form by writing

$$
\begin{array}{ll}
\xi=n / a, & f=a x(\xi), \\
q=a / a m, & w=w I+g(\xi) \Delta w,
\end{array}
$$

where we suppose $g$ is exponentially small at the finner $(\xi-\infty)$ edge of the boundary layer, and is untty at the outer $(\xi=+\infty)$ edge. It should be realized: that $x, w_{I}, \Delta w$ and 8 will generally depend on position on $S$. To the first two orders, however, this dependence only occurs parametrically in the solution, and will therefore be suppressed. Writing

$$
x=x_{0}(\xi)+a x_{1}(\xi)+\ldots .
$$

substituting into (15), and equating like powers of $a$, we obtain

$$
\begin{aligned}
& d^{2} x_{0} / d \xi^{2}-q^{2} g(\xi) x_{0}=0, \\
& d^{2} x_{1} / d \xi^{2}-q^{2} g(\xi) x_{1}=-\left(c_{1}^{-1}+c_{2}^{-1}\right) d x_{0} / d \xi_{,}
\end{aligned}
$$

where $C_{1}$ and $C_{2}$ are the principal radil of curvature of $s$ at the point concerned. Since $f_{\text {II }}$ is identically zero, the solutions to (26) and (27) must obey

$$
x_{0}+0, \quad x_{1}+0, \text { for } \xi++\infty \text {. }
$$

Successful matching to the interior solution, $f_{I}$, requires

$$
x_{0} \sim\left(\partial \varepsilon_{I} / \partial_{n}\right)_{S} \xi, \quad x_{1} \sim-\frac{1}{2}\left(c_{1}^{-1}+c_{2}^{-1}\right)\left(\partial f_{I} / \partial_{n}\right)_{S} \xi^{2}, \text { for } \xi \rightarrow-\infty \text {. }
$$

Explicit solutions can generally be obtalned only by numerical means. They obey integral conditions which we will later find useful (\$4):

$$
\begin{aligned}
& \frac{1}{q^{2}}\left(\frac{\partial f I}{\partial x}\right)_{s}^{2}=\int_{-\infty}^{\infty} x_{0}^{2} \frac{d \xi}{d \xi} d \xi, \\
& -\frac{2}{q^{2}}\left(\frac{1}{c_{1}}+\frac{1}{c_{2}}\right) \mathscr{f}_{-\infty}^{\infty}\left(\frac{d x_{0}}{d \xi}\right)^{2} d \xi=\int_{-\infty}^{\infty} 2 x_{0} x_{1} \frac{d g}{d \xi} d \xi_{,}
\end{aligned}
$$

where the bar through the integral sign signifies that the convergent part of the integral is taken. Despite appearances, this integral is negative. 
The Integral relationships (30) and (31) may be interpreted in the light of the Grant tensor (13). The terms on the left give the main parts of the leap of $\sigma_{\mathrm{nn}}$ across the boundary layer; the terms on the right give the corresponding integrated effects of the external force $f^{2} \vec{v}_{\mathrm{w}}$ balancing them. The dominant term, given on the left of (30), arises from the 'pressure' of the particle tryling to escape the well. The next largest term, shown on the left of (31), clearly has the form of a quantum surface stress, with

$$
T_{\mathrm{m}}=\frac{2 a A \mathrm{w}}{\mathrm{q}^{2}} f_{-\infty}^{\infty}\left(\mathrm{a} \frac{\mathrm{dx}}{\mathrm{d} \xi}\right)^{2} \mathrm{~d} \xi
$$

as coefficent of surface tension. It is easy to make an estimate of $T_{m}$. If we take $g$ to be a unit step function, we find from (26) that

$$
T_{\mathrm{II}} \div-a_{\mathrm{m}}^{3} \Delta \mathrm{w}\left(\partial \mathrm{f}_{\mathrm{I}} / \partial \mathrm{z}\right)_{\mathrm{S}}^{2}
$$

Passing from 'hydrostatics' to 'hydrodynamles' by abandoning (14), we see from (8) that another type of healing phenonmenon will occur when $u$ is large. A particularly significant case occurs at a vortex Iine where, by (11), $u$ is of order $\mathrm{nh} / \mathrm{mw}$, at small distances, $\tilde{\omega}$, from the vortex axis. It follows from (8) and (9) that $\rho$ is of order $\tilde{\omega}^{2 n}$ for $\tilde{\omega} \rightarrow 0$. The fact that $\rho$ is zero on the axis itself means that a closed vortex ring, or a vortex 1 ine terminating on boundarles $(w=\infty)$, will transform an otherwlse simply-connected container into a multiply-connected domain, $R$, so justifying a posterlori the application of (II). Dnilke the healing at a wall considered earlier, the depression of $p$ at the vortex axis occurs over distances comparable with the scale of the container. The correspoading vortices in the condensate discussed in 53 hive cores confined to much smaller distances from their axes.

Before concluding this section we make one remark, obvious perhaps, but relevant to 54 . When the particle is trapped in a potentlal well with moving walls $[w=w(\vec{x}, t)], \phi$ is necessarily non-zero and $\rho$ is time-dependent. Nevertheless, provided the time-scales over which $w$ changes are large compared with the reciprocal of the quantum frequency $\mathrm{h} / \mathrm{mb}^{2}$, we can regard the fluid as being in a quasi-hydrostatic state, ignore the time derivative in (I), and treat $t$ in $w$ parametrically. In quantum language, the Born-Oppenheimer approximation is said to apply.

\section{THE CONDEASATE MODEL}

We now consider an assembly of $N$ identical particles (bosons) of mass $M$ in a potential field W(J). If the particles did not interact, the wavefunction for the system could be written down as a syumetrlzed product of the $N$ oneparticle wavefunctione, $\Psi(\dot{\alpha}, t)$, obeying (11) wth $W$ and $M$ replacing $w$ and $m$. It would be probably more comventent, however, to replace the normalization condition (10) by

$$
\rho|\Psi|^{2} \mathrm{~d} \overrightarrow{\mathrm{Z}}=\mathrm{N}, \text { or } \rho_{\rho \mathrm{d} \overrightarrow{\mathrm{X}}}=\rho_{\infty} \mathrm{V} \text {, }
$$

where $\nabla$ is the volume of the system and $\rho_{\infty}=M N / v$. The resulting theory is well-understood, and contalns features that frutifully represent hellum near absolute zero8. It may be seen from (18), however, that the ground state for, 8ay, the potential well (16) would be one in which all the particles would be at the origin, with high probability. To eliminate this unphysical behavior, the imperfect bose condensate has been devised. A short-range repulsive potential $V\left(\bar{X}-\dot{X}^{\prime}\right)$ is introduced in an ad hoc way, and to $W$ the potential

$$
S V\left(\vec{x}-\vec{x}^{-}\right)\left|\Psi\left(\vec{x}^{\prime}\right)\right|^{2} d \vec{x}
$$


is added which increases as the density of nearby bosons increases. The simplest case arises when $V$ is taken to be

$$
v(\vec{x}-\vec{x})=v_{0} \delta\left(\vec{x}-\vec{x}^{\prime}\right) \text {. }
$$

Equation (1) is then replaced by

$$
\text { In } \partial \Psi / \partial t=-\left(\pi^{2} / 2 M\right) \nabla^{2} \Psi+\left\langle\nabla_{0}|\Psi|^{2}+W\right) \Psi \text {. }
$$

A fuller and more satisfactory derfvation of (37) may be found elsewhere 3,4 . It is of some interest that non-11near Schrobdinger equations of the form (37) have been the subject of close scrutiny in recent years in non-quantal contexts, particularly in theories of weak non-inear waves and stability 9 . The Madelung transformation

$$
\Psi=\operatorname{Fexp}(\mathrm{IM} \Phi / \mathrm{h}) \text {, }
$$

follows the course of $\$ 2$ with minor changes. Most slgnificant is the addition of a 'gas pressure',

$$
p=\left(\nabla_{0} / 2 M^{2}\right) \rho^{2} \text {, }
$$

which (multiplied by $\delta_{i j}$ ) should be included in the stress tensor (13). Thus (8) is replaced by

$$
\frac{\partial \phi}{\partial t}+\frac{1}{2} \vec{u}^{2}+\frac{W}{M}+\frac{2 p}{\rho}+\mu=0 .
$$

The presence of the repulsion, $\nabla_{0}$, and its associated gas pressure restores a number of physical effects absent in 52. The tendency towards condensation is eliminated for all sufficiently large systems. To see this, return to the hydrostatic theory of $\$ 2$ and the potential well (16). The spherically symetrlc $(l=0)$ ground state now obeys

$$
\frac{d^{2} F}{d r^{2}}+\frac{2}{r} \frac{d F}{d r}=\frac{2 M}{\pi 2}(E-W) F-\frac{2 M N_{O}}{\pi^{2}} F^{3}
$$

If $W_{0}$ and $\Delta W$ are both large compared with $h^{2} / \mathrm{Mb}^{2},(41)$ gives everywhere except near the surface, $S$, of the well

$$
\rho=\rho_{\infty}=\mathrm{MF}_{\mathrm{T}}{ }^{2}=\mathrm{MN} / \mathrm{v} \text {, }
$$

the corresponding one-particle energy being given by

$$
E-W_{I}=p_{\infty} \nabla_{0} / M .
$$

The fluid is spread out uniformly in the well.

Near $S$ the derlvatives of $F$ become large, and the constant solution (42) breaks down. We may follow the argument of 52 . Introducing a new bealing length

$$
a=x\left(2 p_{\infty} \nabla_{0}\right)^{-1 / 2}
$$

writing

$$
\xi=n / a, \quad F=X(\xi), W=w_{I}+\left(0_{\infty} \nabla_{0} / M\right) G(\xi),
$$

where $G(\xi)$ is exponentlally swall for $\xi \rightarrow-\infty$, expanding $X$ as

$$
x(\xi)=x_{0}(\xi)+a x_{1}(\xi)+\ldots \text {. }
$$

subst1tuting Into (15), and equating like powers of a, we obtain

$$
d^{2} x_{0} / d \xi^{2}-\left[G(\xi)-1+\left(x_{0} / F_{I}\right)^{2}\right] x_{0}=0 \text {, }
$$




$$
d^{2} x_{1} / d \xi^{2}-\left[G(\xi)-1+3\left(x_{0} / F_{I}\right)^{2}\right] x_{1}=-\left(c_{1}{ }^{-1}+c_{2}^{-1}\right) d x_{0} / d \xi .
$$

Matching at the edges of the boundary layer requires

$$
\begin{aligned}
& x_{0}+0, \quad x_{1} \rightarrow 0, \quad \xi \rightarrow+\infty ; \\
& \mathrm{X}_{0} \rightarrow \mathrm{F}_{I}, \mathrm{X}_{1}+0, \quad \xi \rightarrow-\infty .
\end{aligned}
$$

Again, explicit solutions generally require numertcal integrations, though useful integral relations may be established, for instance

$$
\begin{aligned}
& \frac{1}{2} F_{I}^{2}=I_{-\infty}^{\infty} x_{0}^{2} \frac{d G}{d \xi} d \xi, \\
& \left.-2\left(\frac{1}{c_{1}}+\frac{1}{c_{2}}\right) f_{-\infty}^{\infty} \frac{d x_{0}}{d \xi}\right)^{2} d \xi=-\infty 2 x_{0} x_{1} \frac{d G}{d \xi} d \xi .
\end{aligned}
$$

Once more, the dominant contribution to the leap in stress across the healing layer arises from the interfor solution, although now it is the gas pressure and not the quantum pressure that is mainly responstble. Again, the next largest term can be interpreted as a quantum surface tension, with positive coefficient

$$
T_{M}=2 a\left(\frac{\rho_{\infty} \nabla_{0}}{M}\right) r_{-\infty}^{\infty}\left(\frac{d x_{0}}{d \xi}\right)^{2} d \xi \text {. }
$$

As before, $\mathrm{T}_{\mathrm{M}}$ may be estimated 3 from a simple model of $\mathrm{z}$. If we suppose that $G$ is a step function of infinite helght $(\Delta W=\infty),(46)$ may be solved as $X_{0}=F_{I} \tanh (-\xi / \sqrt{2})$, and $(53)$ gives

$T_{M}=\sqrt{2} \pi^{2} 0 / 3 M^{2} a$.

Pasalng again from 'hydrostatics' to 'hydrodynamlcs', we note that the gas pressure can supply the restoring force necessary for compressional waves. Perturbing about the static solution (41), we readily find that long wave-length sound propagates at the velocity

$$
c=\sqrt{ }(\mathrm{dp} / \mathrm{do})=\sqrt{ }(2 \mathrm{p} / \mathrm{o})=\pi / \mathrm{Ma} / 2 \text {. }
$$

At wavelengths of order $a$ and smaller, the quantum pressure increases the phase speed, decreases the group velocity, and introduces weak dispersion.

Vortex lines be studied as in \$2. Unlike their classical counterparts, the cores of these vortices do not have sharply defined surfaces separating regions of zero and non-zero vorticlty. All the vorticity they contain is concentrated as $\delta$-functions on their axes. Such a vortex, if classical, would have infinite self-energy. Here, however, the density decreases over the characterlstic distance $a$ as the axis is approached, so ensuring a fintte tension. The depletion of flutd in the core makes the vortex resemble the classical hollow core model. The permanence of vortex rings implied by the Relvin-Belmholtz theoren makes them excellent candidates for quasi-particle models, so reviving in a novel context the ideas underlying the vortex atoms proposed by Relvin in the nineteenth century.

\section{STRDCTURE OF THE NEGATIVE ION}

It is possible $3,10-12$ to account with relative ease for many features of the negative 1on by combining the methods of $\$ \$ 2$ and 3 above. We use the theory of 51 to represent the electron, regarding as the potential created by the surrounding condensate; we apply the formalism of $\$ 2$ to the 
exterlor of the bubble, taking for $w$ the potential of the electron. Wore explicitily, we introduce the energy,

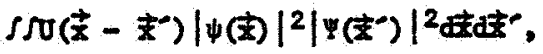

representing the repulgion of an electron at $\vec{x}$ and $a$ boson at $\vec{x}^{*}$. Takting agaln the olmplest case of a s-function interaction

$$
v\left(\vec{x}-\vec{x}^{\prime}\right)=v_{0} \delta\left(\vec{x}-\vec{x}^{\prime}\right) \text {, }
$$

we then have

$$
\begin{aligned}
& w(\vec{x})=\int U\left(\vec{x}-\vec{x}^{\prime}\right)|\Psi(\vec{x})|^{2} d \vec{x}=v_{0}|\Psi(\vec{x})|^{2}, \\
& w(\vec{x})=\int U\left(\vec{x}-\vec{x}^{\prime}\right)|\psi(\vec{x})|^{2} d \vec{x}=v_{0}|\psi(\vec{x})|^{2}
\end{aligned}
$$

By $(10)$ and $(58), \Delta w=U_{0 P a d} / M$ so that by (22), (23) and (44), $q^{2}=m D_{0} / N_{0}$. Equations (1) and (37) become coupled:

$$
\begin{aligned}
& \operatorname{Ir} \partial \psi / \partial t=-\left(\hbar^{2} / 2 m\right) \nabla^{2} \psi+\delta_{0}|\Psi|^{2} \psi, \\
& \operatorname{In} \partial \psi / \partial t=-\left(\hbar^{2} / 2 M\right) \nabla^{2} \Psi+\left(v_{0}|\Psi|^{2}+\delta_{0}|\psi|^{2}\right) \Psi .
\end{aligned}
$$

As In 52, we define the electronic surface, $S$, of the bubble by the zero of $\psi$.

The key to a simple 'bydrostatic' solution of (60) and (61) lies in the fact, which we can verlfy a posterlorl, that the radius, $b$, of the bubble is large compared with a and $a_{m}, 80$ that the boundary layer methods of $5 \$ 2$ and 3 can be used with minor emendations. We must not forget however that, since the roles of interfor and exterfor of the bubble have been exchanged for the condensate, the sign of $\xi$ in $\$ 3$ must be reversed. The mainstream value of $F$, denoted in 53 by $F_{I}$ is now written $F_{S}$.

To leading order, we set $g(\xi)=x_{0}{ }^{2} / F_{S}{ }^{2}$ and $G(\xi)=\left(a^{2} U_{0} / F S^{2} V_{0}\right) x_{0}{ }^{2}$ in (26) and (47), as (58) and (59) require. The integral relations (30) and (5I) may then be combined to give

$$
\frac{a^{2}}{q^{2}}\left(\frac{\partial f}{\partial n}\right)_{s}^{2}-\frac{v_{0}}{2 U_{0}} F_{s}{ }^{2}=\frac{a^{2} U_{n}}{F_{s}^{2}}\left[x_{0}{ }^{2} x_{0}{ }^{2}\right]_{-\infty}^{\infty}=0,
$$

where we have appealed to (28) and (50). To the next order, the forms of 8 and $G$ require reconsideration 12 . In place of $(27)$ and $(48)$ we have

$$
\begin{gathered}
d^{2} x_{1} / d \xi^{2}-\left(q / F_{S}\right)^{2}\left(x_{0}{ }^{2} x_{1}+2 x_{0} x_{0} x_{1}\right)=-\left(c_{1}{ }^{-1}+c_{2}{ }^{-1}\right) d x_{0} / d \xi_{0} \\
d^{2} x_{1} / d \xi^{2}-\left(3 x_{0}{ }^{2} / F_{S}{ }^{2}-1\right) x_{1}-\left(a^{2} d_{0} / F_{S}{ }^{2} v_{0}\right)\left(x_{0}{ }^{2} x_{1}+2 x_{0} x_{0} x_{1}\right)= \\
=-\left(c_{1}{ }^{-1}+c_{2}{ }^{-1}\right) d x_{0} / d \xi_{.}
\end{gathered}
$$

The integral consequences (3I) and (52) are modifled accordingly, and the result (62) is altered to

$$
\frac{a^{2}}{q^{2}}\left(\frac{\partial f}{\partial n}\right)_{S}^{2}-\frac{\nabla_{0}}{2 D_{0}} F_{S}^{2}=2 a\left(\frac{1}{C_{1}}+\frac{1}{C_{2}}\right) f_{-\infty}^{\infty}\left[\frac{a^{2}}{q^{2}}\left(\frac{d x_{0}}{d \xi}\right)^{2}+\frac{\nabla_{0}}{U_{0}}\left(\frac{d X_{0}}{d \xi}\right)^{2}\right] d \xi
$$

which now includes the effects of interfacial tension.

The jump conditions (62) or (65) across the boundary layer suffice to match the mainstream electron solution to the mainstream condensate solution. Applied to the electron bubble, we have by (18)

$$
f=A\left(\sin \lambda_{I} r\right) / \lambda_{I} r
$$


ahowing that $\lambda_{I b}=\pi$. By $(10)$ the solution is normalized to the first two orders 12 in $a / b$, if $A^{2}=\pi / 2 b^{3}$. By (10) and (11) we obtain

$$
b=\left(\frac{\pi u^{2} a^{2}}{m p_{\infty}}\right)^{1 / 5}-\frac{8 M^{2} a}{5 p_{\infty}} f_{-\infty}^{\infty}\left[\frac{1}{m}\left(\frac{d x_{m}}{d \xi}\right)^{2}+\frac{1}{M}\left(\frac{d x_{a}}{d \xi}\right)^{2}\right] d \xi \text {. }
$$

It is not at once clear whether $b$ w111 be decreased by the positive surface tension (53) of the condensate or increased by the negative surface tension (32) of the electron. If we use estimates (33) and (54) however, we see that $\left|\mathrm{T}_{\mathrm{m}}\right| / \mathrm{T}_{\mathrm{M}}$ is of order $\mathrm{q}^{-1}$ and, afnce experiments indicate (\$5) that $q<1$, it appears that the bubble radius should be larger than $\left(\pi M^{2} a^{2} / m 0_{\infty}\right) 1 / 5$. Direct numertcal integrations 12 of $x_{0}$ and $x_{0}$, and evaluation of the integral aeen In (67), suggest that the difference 18 of the order of $a$.

The effects of polarization induced by the electron in the surrounding hellum can be Included by adding 12 to $(56)$ the term

$$
-\left(\tilde{\alpha}^{2} / 8 \pi\right) f f|\psi(\vec{x})|^{2}|\psi(\vec{x})|^{2}|\vec{x}-\vec{x}-|^{-4} d \vec{x} d \vec{x}
$$

where $\tilde{a}$ is the polarizability of the hellum and $e$ is the electrontc charge. This has the effect of contracting the bubble by order $a_{M e}^{2} a^{2} / 4 \pi \pi^{2} b^{3}$. A detalled theory ${ }^{12}$ shows that the reduction is of order $a / 3$ in the practically Interesting cases.

Further complications arise when the dynamics of the bubble are considered, elthough the time-scales of Interest are usually large enough compared with the electronte frequencles to justify the neglect of $\partial \psi / \partial t$ in $(60)$; see 52 . To evaluate the effective hydrodynamic mass, we consider the bubble in ateady wotion $\forall$, at small Mach numbers $\Re=0 / c$. The electronic radius, $b$, of the bubble is Increased by about $5 M^{2} z$ because of the pressure forces associated with the flow of condensate over its surface. It is also made silghtly oblate, with an ellipticity close to $M^{2} / 2$. Ignoring this effect, it is found that the dipolar back-flow created by the lon colncides with that of a hard sphere whose radius, $b_{e}$, is less than the electronte radius, $b$, by one to two heeling lengths:

$$
b_{e}=b-\left(a M / p_{\infty}\right) \underline{\varepsilon}_{-\infty}^{\infty} x_{0}{ }^{2}(\xi) d \xi \text {. }
$$

It is this radius, rather than $b$, that determines the Induced mass of the lon.

Further detalls of the calculations qutlined above may be found in the paper by Roberts and Grant already cited ${ }^{12}$. We conclude this section by breaking new ground. We consider the oscillations of the bubble, their implicatlons for phonon-1on collisions, and the mobility of negative lons at low temperatures. We agatn adopt the boundary layer methods deacribed above but, of course, retain the term $\partial \Psi / \partial t$ in $(61)$, so introduclng a velocity potential, $\phi$, In the condensate. We retain only the dominant part of the boundary layer structure, excluding both surface tension and polarlzation effects. We write,

$$
f=E_{0}+\alpha_{a f}, \quad F=F_{0}+\alpha a F^{-}, \phi-\alpha \phi^{-},
$$

where the suffix o stands for the steady solution obtalned earlier, and the terms in a represent time-dependent perturbations, where $0<a<1$.

It is readily seen from (7) and (40) that $\Phi^{-}$and $F^{-}$both obey the acoustic wave equation

$$
\partial^{2} \Phi^{-} / \partial t^{2}=c^{2} \nabla^{2} \phi^{-}
$$


We could, by following Cell1, Cohen and Zuckerman ${ }^{13}$, examtne solutions in the form of outgoing waves. The elgenfrequencles would be complex, because of the reduction in oscillation amplitude at a polnt fixed in space as the energy of surface motion is radiated to infintty. The corresponding eigenfunction must tend to infintty with $r$ since, the more distant a wave 18 , the earlier it must have left the surface, and the greater the amplitude of surface oscillation must then have been. We will not consider solutions of this type below. We will confine our attention to the scattering problem in which an incoming plane wave travelling in the $\mathrm{z}$-direction

$$
S_{\text {IVC }}^{-}=\exp [1(k z-\omega t)] \text {. }
$$

where $k$ and $\omega=$ ck are real, 18 scattered by the bubble into a set of outgoing waves. We first gim to calculate the scattering amplitude, $h_{\ell}(k)$, of the lth partial wave ${ }^{13}$

$$
h_{\ell}=\frac{1}{1+1 Q_{\ell}}, \quad Q_{\ell}=\frac{k b_{\ell}(k b)+K_{\ell} y_{l}^{-}(k b)}{k b j_{\ell}(k b)+K_{\ell} j_{l}^{-(k b)}},
$$

where $y_{l}(z)$ is the spherical Bessel function of the second $k$ ind and $k_{\ell}$ is the spring constant of the bubble for this mode. We then use ho to compute the differential cross-section ${ }^{14}$ of the bubble

$$
\sigma(k, \theta)=\left.\left.k^{-2}\right|_{\ell=0} ^{\infty}(2 l+1) h_{\ell}(k) P_{n}(\cos \theta)\right|^{2},
$$

and hence the momentum-transfer cross-sect $10 n^{14}$

$$
\sigma_{T}(k)=\int \rho \sigma(k, \theta)(1-\cos \theta) \sin \theta d \theta d x \text {. }
$$

From this we finally evaluate the mobllity, $\mu_{e}$, of the fon from 14

where

$$
\frac{e}{\mu_{e}}=-\frac{\hbar}{6 \pi^{2}} \stackrel{f}{\infty}_{-\infty}^{\infty} \sigma_{T}(k) \frac{\partial n(k)}{\partial k} k_{k}^{4} d k \text {, }
$$

$$
n(k)=[\exp (\text { rak } / \mathrm{KT})-1]^{-1}
$$

Is the density of phonons (in $\vec{k} \vec{k}$-space) at temperature $T$. Here $K$ is Boltzmann's constant.

To determine the spring constants, $\mathrm{K}_{\ell}$, we have to match solutions of (71) across the boundary layer on $S^{-}$, the deformed electronic surface, to the quasi-static solutions (18) of the electron mainstream. We first consider the case $\& \geq 1$. The fact that

$$
\psi=A_{0} J_{0}\left(\lambda_{I} r\right)+\operatorname{caA}^{-} j_{l}\left(\lambda_{I} r\right) Y_{l}(\theta, X) \text {, }
$$

Implles that $S^{+}$has the equation

$$
I=b(\theta, x, t) \equiv b_{0}+a a b^{-}(t) Y_{\ell}(\theta, x) \text {, }
$$

where (using $\lambda_{I} b_{0}=\pi$ )

$$
b \% / b_{0}=j_{l}(\pi) A \% / A_{0} \text {. }
$$

We will continue to refer the boundary layer structure to the unperturbed position, $S_{0}$, of the electronic surface, and not to $S^{+}$. We introduce $x^{-}, X^{-}$ and $\eta^{\circ}$, the boundary layer forms of $f^{\circ}, F^{-}$and $\Phi^{\circ}$, and expand these in ascending powers of a

$$
x^{\prime}=a^{-1} x_{0}^{\prime}+x_{1}^{\prime}+\ldots, \quad x^{-}=a^{-1} x_{0}+x_{1}+\ldots, n^{-}=n_{0}^{-}+a_{n} n^{-}+\ldots
$$


where the coefficients shown depend on $t$ and $\xi=\left(r-b_{0}\right) / a$ and parametrically on $\theta$ and $x$. We substitute these into the boundary layer forms of (60) and (61) which, in the Madelung framework, give to the first two orders

$$
\begin{aligned}
& \partial^{2} x / \partial \xi^{2}-\left(g / F_{S}\right)^{2}\left(x_{0}^{2} x^{\circ}+2 x_{0} x_{0} x^{0}\right)=-(2 a / b) \partial x^{-} / \partial \xi \text {, } \\
& \partial^{2} X^{-} / \partial \xi^{2}-\left(3 x_{0}^{2} / F_{S}^{2}-1\right) X^{0}-\left(a^{2} v_{0} / F_{S}^{2} v_{0}\right)\left(x_{0}{ }^{2} X^{-}+2 x_{0} x_{0} x^{\prime}\right)= \\
& =-(2 a / b) \partial X / \partial \xi+\left(M / F_{S}^{2} \nabla_{0}\right) X_{0} \partial n-/ \partial t \text {, } \\
& x_{0} \partial^{2} \eta / \partial \xi^{2}+2\left(d x_{0} / d \xi\right) \partial \eta / \partial \xi=-(2 a / b) x_{0} \partial \eta / \partial \xi-2 a^{2} \partial x-/ \partial t \text {. }
\end{aligned}
$$

It seems clear from (84) that $x_{0}^{2} \partial n_{0} / \partial \xi$ is Independent of $\xi$ and, stace there is no zet condensate flow through the boundary layer at any point, that constant must be zero. Thus no takes throughout the boundary layer the mainstream value of $q$, evaluated on $S_{0}$. The right-hand side of (83) does not contribute to leading order in a, and (82) and (83) may be solved to give

$$
x_{0}^{\prime}=\zeta d x_{0} / d \xi, \quad x_{0}^{\prime}=\zeta d x_{0} / d \xi \text {, }
$$

where $\zeta$ is independent of $\xi$. These forms represent a net displacement of the equilibrium boundary layer from $S_{0}$ to $S^{\prime}$, without change of form; we conclude that $\zeta=-b Y_{\ell}$.

In proceeding to the next order we note that, since the veloctty of sound (55) is of order $1 / \mathrm{a}$ the time derivatives in (83) and (84) now contribute. In fact, excluding again a net flux of condensate through the boundary layer, (84) shows that $\partial n_{1} / \partial \xi$ takes the value $-a^{2} \partial \xi / \partial t$ throughout, and in particular on the outer edge $(\xi=\infty)$ of the boundary layer. It follows that

$$
a \partial b^{\prime} / \partial t=\left(\partial \phi^{-} / \partial x\right) S,
$$

an equation with an obvious interpretation. The equations (82) and (83) again admit an integral, namely

$$
\frac{M x_{0}^{2}}{2 \bar{U}_{0} F_{S}^{2}} \frac{\partial n_{0}{ }^{-}}{\partial t}=\frac{a^{2}}{q^{2}}\left[\frac{d x_{0}}{d \xi} \frac{\partial x_{1}^{-}}{\partial \xi}-x_{1}^{-}-\frac{d^{2} x_{0}}{d \xi^{2}}\right]_{-\infty}^{\xi}+\frac{V_{0}}{U_{0}}\left[\frac{d x_{0}}{d \xi} \frac{\partial^{2} x_{1}^{\prime}}{\partial \xi^{2}}-x_{1}^{-} \frac{d^{2} x_{0}}{d \xi^{2}}\right]_{-\infty}^{\xi}
$$

On taking the limit $\xi \rightarrow+\infty$, and using (18) to evaluate the contributions from the lower 1imits, we find

$$
\partial \Phi_{S} \cdot \partial t=c^{2} \mathbb{R}_{\ell} a b \div / b_{0} \text {, }
$$

where

$$
K_{\ell}=\left\{\begin{array}{l}
5 / 2, \text { if } \ell=0 ; \\
1-\ell+\pi J_{\ell-1}(\pi) / J_{\ell}(\pi), \text { if } \ell \geq 1 .
\end{array}\right.
$$

The numerical values of $K_{\ell}$ for the first 20 values of $\&$ are given in table $I$. That of $k_{0}$ was obtained from an analysis too simflar in spirit to the one just described to be repeated here. It way be noted that $R_{1}$ is zero, representing the fact that the bubble is neutrally stable to a uniform displacement. Equations (86) and (88) are applied on $S_{0}$, and provide the boundary conditions to which solutions of (71) must be subjected.

We developed a program for an Hewlett-Packard 9820 A desk computer to evaluate $\sigma_{\mathrm{T}}(\mathrm{k})$ and $\mu_{\mathrm{e}}$ from an arbitrary set of the spring constants. The 
results were tested with the values $\left(K_{0}=0.23474 ; K_{1}=0 ; K_{2}=0.45045\right.$; $\left.\bar{K}_{n}=0, n>2\right)$ used by Baym, Barrera and Pethick ${ }^{14}$, and good agreement was obtalned. The programe was then used to generate the results shown in Figures 1 and 2. The effect of truncating sertes (74) at $l-2$ and $\ell=19$ are shown in both cases. The prominent peak in $\sigma_{T}(k)$ seen in Figure 1 is due to a d-wave resonance $(l=2)$. A new minor peak is added every time $\&$ is incremented by 1 . The curve appears to approach 1ts geometrical value 15 $\left(\sigma_{T} / 4 \pi b_{0}{ }^{2} \rightarrow 1 / 2\right)$ with an oscillation of amplitude $\left(k_{0}\right)^{-2 / 3}$ and period $2^{-1 / 3}$. In FIgure 2, we see $\mu_{e} T^{3}$ plotted in units of $L_{0}$, as a function of $T$ measured in units of $T_{0}$ where

$$
T_{0}=x c / b_{0} R, \quad L_{0}=3 \pi e \kappa^{2} c^{3} / 2 b_{0} R .
$$

TABLE 1

Spring Constants

$\begin{array}{lrll}\ell & K_{\ell} & l & Z_{\ell} \\ 0 & 2.500000000 & 10 & 11.56327795 \\ 1 & 0.000000000 & 11 & 12.59928101 \\ 2 & 2.289868134 & 12 & 13.62973970 \\ 3 & 3.771253431 & 13 & 14.65585350 \\ 4 & 5.032253885 & 14 & 15.67849696 \\ 5 & 6.198547165 & 15 & 16.69832328 \\ 6 & 7.314641577 & 16 & 17.71583055 \\ 7 & 8.400646541 & 17 & 18.73140537 \\ 8 & 9.467085072 & 18 & 19.74535255 \\ 9 & 10.520037400 & 19 & 20.75791573\end{array}$

\section{EXPERTMENTAI COMPARISONS}

The condensate model of helium II is essentially a theory having only one disposable parameter, namely the pseudo-potential, $\nabla$, or equivalently the healing length, a. It is natural to seek to choose this so that theory and observation are in optimum accord. Clearly a choice of a made to fit one physical phemomenon well is likely to conflict with others, and an overall consistency with the experimental facts is not to be anticipated. One notes particularly that, since the condensate is a gas obeying the equation of state (39), we should not expect the theory to perform well at the vapor pressure.

One can obtain an estimate of $\nabla=4 \pi \mathrm{d}^{2} / M$ from measurements of the atomic diameter, $d$, by $\alpha$-particle scattering expertments. Values of $d$ of about $2.7 \AA$ have been found. If $\rho_{\infty}$ is $0.145 \mathrm{~g} / \mathrm{cm}^{3}$, the heallng length would be $0.82 \AA$, leading to too small a velocity of sound. One popular procedure has been to extract a from accurate experimental determinations of the relation between the velocity and energy of circular vortex rings. This had led to estimates of a $1.28 \AA$, giving much too small a value of $c$. The reliability of the approach can, however, be questloned. One would have expected a to decrease with increasing pressure, but the reverse seems to be true ${ }^{16}$. It is now belleved 17 that the core of a superfluid vortex is the seat of excitations (normal fluid), and that the surface of a vortex core marks the distance from the axis at which the Landau critical velocity is reached, rather than a quantum healing distance; such a bellef is consistent with the increase of a with $\rho_{\infty}$. 
Pexhaps the most satisfactory way of estimating a is through the velocity of sound (55). To give an example, if we take $c=238 \mathrm{~m} / \mathrm{g}$, we obtain $a \div 0.47 \AA$ and for $\rho_{\infty}=0.145 \mathrm{~g} / \mathrm{cm}^{3}$ we find that $V_{0}+1.710^{-37} \mathrm{~g} \mathrm{~cm} / \mathrm{s}^{2}$, a value admittediy three times larger than the scattering expertments suggest, and

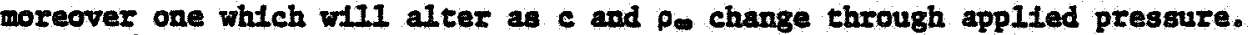
Neverthéless, by using $c$ to determtne $a$, we obtatn a coefflclent of surface tenslon, $T_{M}$, from estfmate (54) of $0.37 \mathrm{~g} / \mathrm{s}^{2}$, which $1 \mathrm{~s}$ in good agreement whth the experimental value of $0.34 \mathrm{~g} / \mathrm{s}^{2}$ at low temperatures.

Turning to the bubble, we see that, In the first approxtmation, the theory does not require a knowledge of the pseudo-potential, $U_{0}$, elther for 1 ts equiIibrium structure or for lts oscillation spectrum. The radius, $\left(\pi \mathrm{K}^{2} \mathrm{a}^{2} / \mathrm{mo}\right)^{1 / 5}$, predicted by the first approximation ts somewhat small, $11.8 \AA$ using the values quoted above. Since $c$ and po Increase with Increasing pressure, $p$, this radius decreases with increasing presaure, although someshat more slowiy than experiments indicate. The bubble radius is increased when the effects of surface tenston are added. Unfortunately, Grant and Roberts 12 did not examine values of a as small as $0.47 \AA$, so that the value of the integral appearing in (67) is not known. Using our earlier estimates, however, it appears that $b$ will be increased to about $13.3 \AA$ by surface tension effects. Table 2 gives $\mu_{\mathrm{e}} \mathrm{T}^{3}$ for a few values of $T$ for both the $l=2$ and the $l=19$ truncattons, and for values of $b$ of $11.8 \AA, 13.3 \AA$ and $16.0 \AA$. At the $l=2$ level of truncation, there $1 \mathrm{~s}$ a clear tendency for $\mu_{\mathrm{e}} \mathrm{T}^{3}$ to approach a limiting value, of about $36 \mathrm{~cm}^{2} \mathrm{k}^{3} \mathrm{vs}_{\mathrm{s}}$ in the case of the $16 \AA$ bubble, as $T$ increases. The explanation of this behavior was provided by Baym, Barrera and Pethtck14 in terms of the shape of the d-wave resonance of Fig. 1. Not surprisingly in view of the very different form of or obtained at $l=19$ truncation, the constancy of $\mu_{\mathrm{e}} \mathrm{T}^{3}$ is not as marked at this level. Nevertheless, the values shown for $l=19$ in Table 1 are not ridiculously fax from the experimental value 18 of about $32.5 \mathrm{~cm}^{2} \mathrm{~K} 3 \mathrm{~s}$ in the range of I tn which Baym, Barrera and Pethick measured the success of the work.

When we take the theory of the bubble to the second approximation, a new disposable parameter enters, namely the psuedo-potential, $0_{0}$, or equivalently $q=a / a_{m}$ a relation we can also write as $q^{2}=\mathrm{mU}_{0} / \mathrm{MV}_{0}$. Roughly speaking $q$, as the ratio of the two healing lengths, measures the relative penetration of the condensate wavefunction into the cavity to the penetration of the electron wavefunction into the condensate. If $q$ were zero, it would be legitimate to treat the condensate as an abrupt edge and only consider the electronic boundary layer of 52 . At first sight it might appear that, since $q^{2}$ is proportional to $m / M=1.3710^{-4}$ it would be admissible to follow Celli, Cohen and Zuckerman 13 in taking this view. The indications are, however, that $\nabla_{0} / \nabla_{0}$ is large. Scattering experiments give an electron-helfum scattering. Iength, $\lambda$, of about $0.60 \AA$, Implying that $0_{0}=2 \pi \lambda \pi^{2} / \mathrm{m}$ is about $4.610^{-35} \mathrm{~g} \mathrm{~cm} / \mathrm{s}^{2}$. Taken with the expertmental value of $5.710^{-38} \mathrm{~g} \mathrm{~cm} / \mathrm{s}^{2}$ for $\nabla_{0}$, we obtain $\nabla_{0} / \nabla_{0} \div 810$ and $q$ 0.33. It would be interesting to see whether the effect of restoring $q$ to the Celli-Cohen-Zuckerman theory would have serlous repercussions. The indications are that $1 t$ would not.

The neglect of $q$ in the condensate theory described here would eliminate the condensate surface tension, $T_{M}$, and transforn the interfactal boundary layer into the structure considered in $\$ 2$. The associated negative surface tenston; $T_{m}$, would tend to expand the bubble, an effect confirmed by the calculations of Grant and Roberts ${ }^{12}$. All influences of interfacial tension are, however, of second order in the condensate theory. In the approach of Cell1, Cohen and Zuckerman 13 , the interfactal tension, $\sigma$, is a first order effect. 
These authors regarded $\sigma$ as a disposable parameter that could be legitimately chosen to fit the observed bubble radius at the applied pressure of Interest. It is easy to verify that the interfactal tension they require is positive and, particularly at higher pressures, several times larger than the condensate aurface tension, $I_{\text {, }}$ considered earlier. It may be wondered why, wh this sign difference, the bubble radil they obtain are, being in perfect agreement with experiment, larger than those obtained from the condensate. The answer is to be found in (39). The condensate is a gas and, to obtain agreement with the observed helium densitles, it is necessary to choose a large $\nabla_{0}$, leading to pressures (39) of the order of 40 atmospheres. In contrast, the Celli, Cohen and zuckerman theory treats the hellum as a classical compressible fluid, not contalning the pressure (39), and to avold large bubble radil at the vapor pressure, a positive interfacial tension is needed. As we have stated above, we regard (39) as an artificial construct of the theory, not to be Identified with the applfed pressure, and base our comparison with experiment on density and velocity of sound data.

As Schwarz15 observes, if the spring constants were regarded as disposable parameters, there would be no difficulty in reproductng any ion mobility data prectsely. It appears that even the added flexibility given to the theory by the ad hoc interfacial tension, $\sigma$, already permits an excellent account of the mobilities. Schwarz 15 has shown that, for their spring constants, the constancy of $\mu_{\mathrm{e}} \mathrm{T}^{3}$ in the range of $\mathrm{T}$ of interest is not lost when the truncation level is Increased as it is in ours. In comparing our theory with theirs, one must be perplexed by the substantial difference in the spring constants and in the shape of the mobility curve (labelled ' $l=19^{\prime}$ ' in Fig. 2 ). He must wonder if, in the disappointing form of that curve on the present theory, and in the sensitivity of the mobllity itself to the healing length [as evinced by the c-dependence of $(90)]$, the condensate theory has not met 1 ta most severe test to date. He may also speculate on the physical basis of the ad hoc interfactal tension required by the other approach to survive its trial by experiment, and also whether the effects of roton-10n collisions at the higher temperatures have been underestimated.

TABLE 2

Ion Mobilities, $\mu_{e}$

( $T$ in degrees $R, H^{T^{3}}$ in units of $\mathrm{Cm}^{2} \mathrm{~K}^{3} \mathrm{Vs}$ )

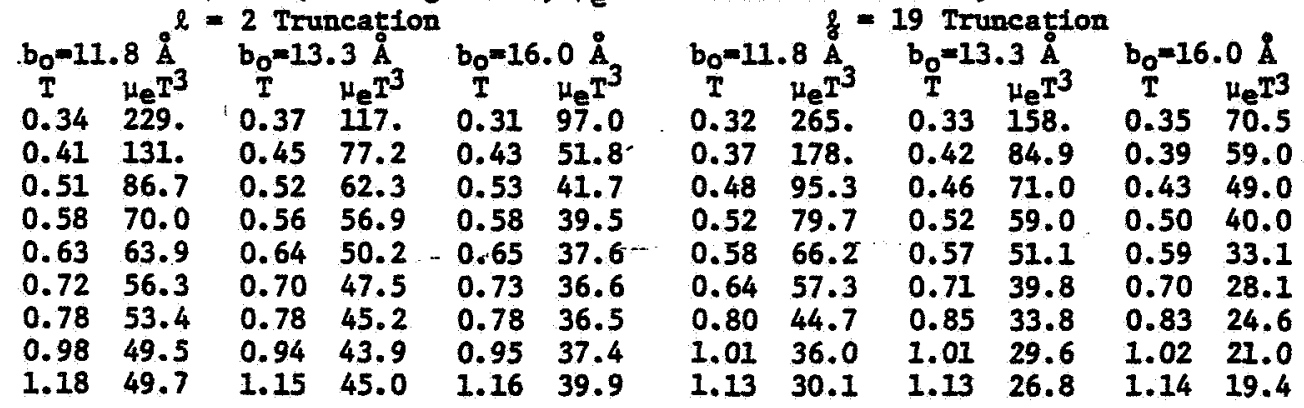


ACXNOWLEDGEMENIT

I an extremely grateful to Professor Russell $\mathrm{J}$. Donnelly for his criticism of this work, and particularly for his contributions to 35 . The work described was supported by the Air Force Office of Sclentiflc Research under grant AFAFOSR-71-1999 and by the National Sclence Foundation under grant NSF GH 35898 .

\section{REFERENCES}

1. R. J. Donnelly, "Experimental Superfluidity," Chicago University Press, Chicago (1967).

2. A. L. Fetter, "The Physics of Liquid and Solld Belium," ed. K. H. Bennemann and J. B. Retterson, Wiley, New York (1975).

3. E. P. Gross, "Quantum Fluids," ed. D. F. Brewer, North Holland, Amsterdam (1966).

4. A. L. Fetter and J. D. Walecka, "Quantum Theory of Many Particle Systems," McGraw H111, New York (1971).

5. E. Madelung, 2. fur Phys. 40, 322 (1927).

6. J. Grant, Ph.D. Thesis, University of Nercastle (1972).

7. J. Grant, J. Phys. A, 6, L151 (1973).

8. S. Putterman, "Superfluld Hydrodynamics," North Holland, Amsterdam (1974).

9. L. M. Hocking and K. Stewartson, Mathemat1ka 18, 219 (1971).

10. R. C. Clark, Phys. Lett. 16, 42 (1965).

11. R. C. Clark, "Superfluld Helium," ed. J. F. Allen, North Holland, Amgterdan (1966).

12. J. Grant and P. H. Roberts, J. Phys. A., I, 260 (1974).

13. V. Cell1, M. H. Cohen and M. J. Zuckerman, Phys. Rev. 173, 253 (1968).

14. G. Baym, R. E. Barrera and C. J. Pethlck, Phys. Rev. Lett. 22, 20 (1969).

15. N. F. Mott and H. S. W. Massey, "The Theory of Atomic Collisions," Third Edition, Clarendon Press, Oxford (1965).

16. M. Steingart and W. I. Glaberson, Phys. Rev. A., 5, 985 (1972).

17. W. I. Glaberson, J. Low Temp. Phys. 4, 289 (1969).

18. R.W. Schwerz and R. W. Stark, Phys. Rev. Lett. 21, 967 (1968).

19. R. W. Schwarz, Phys. Rev. A., 6, 1958 (1972). 


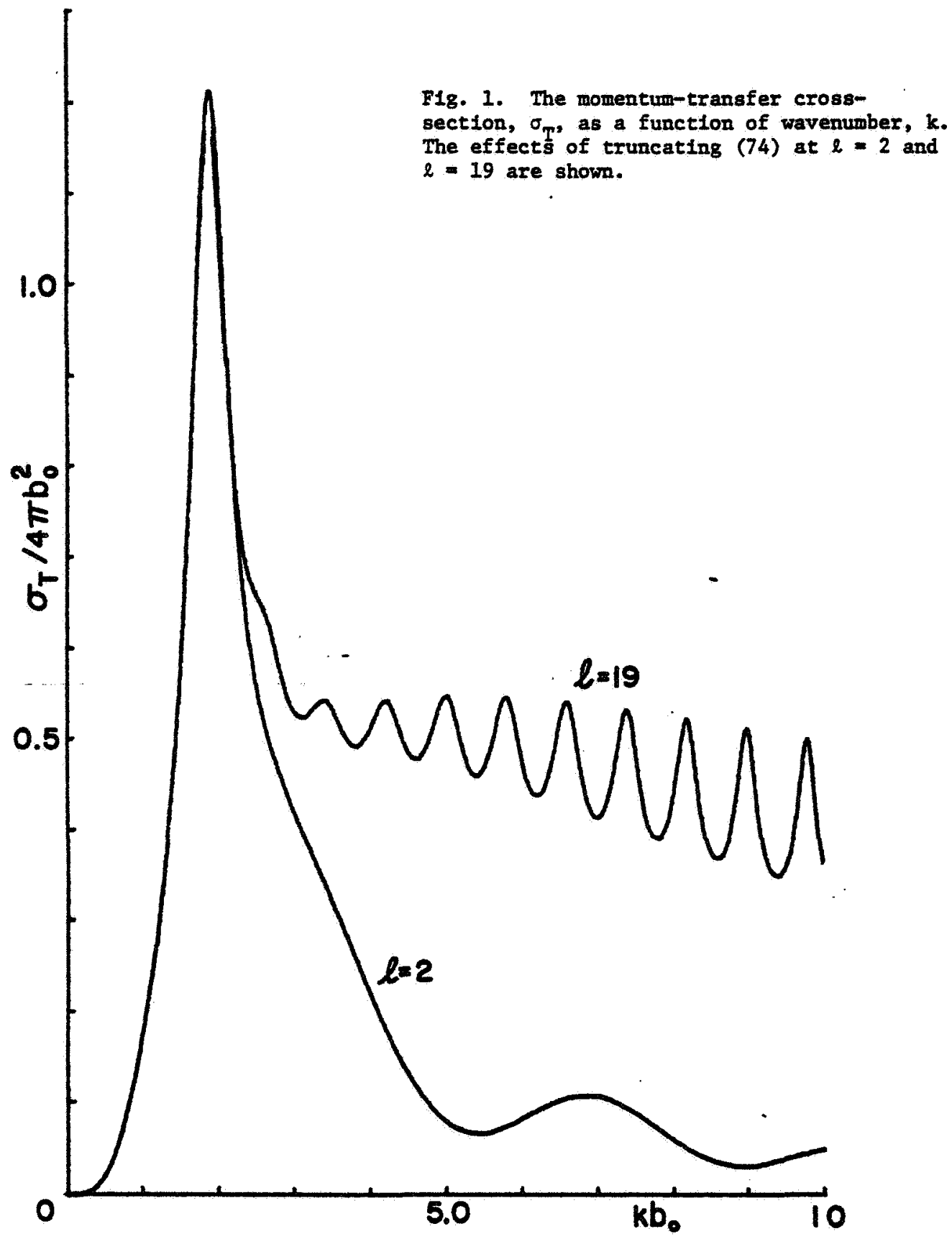




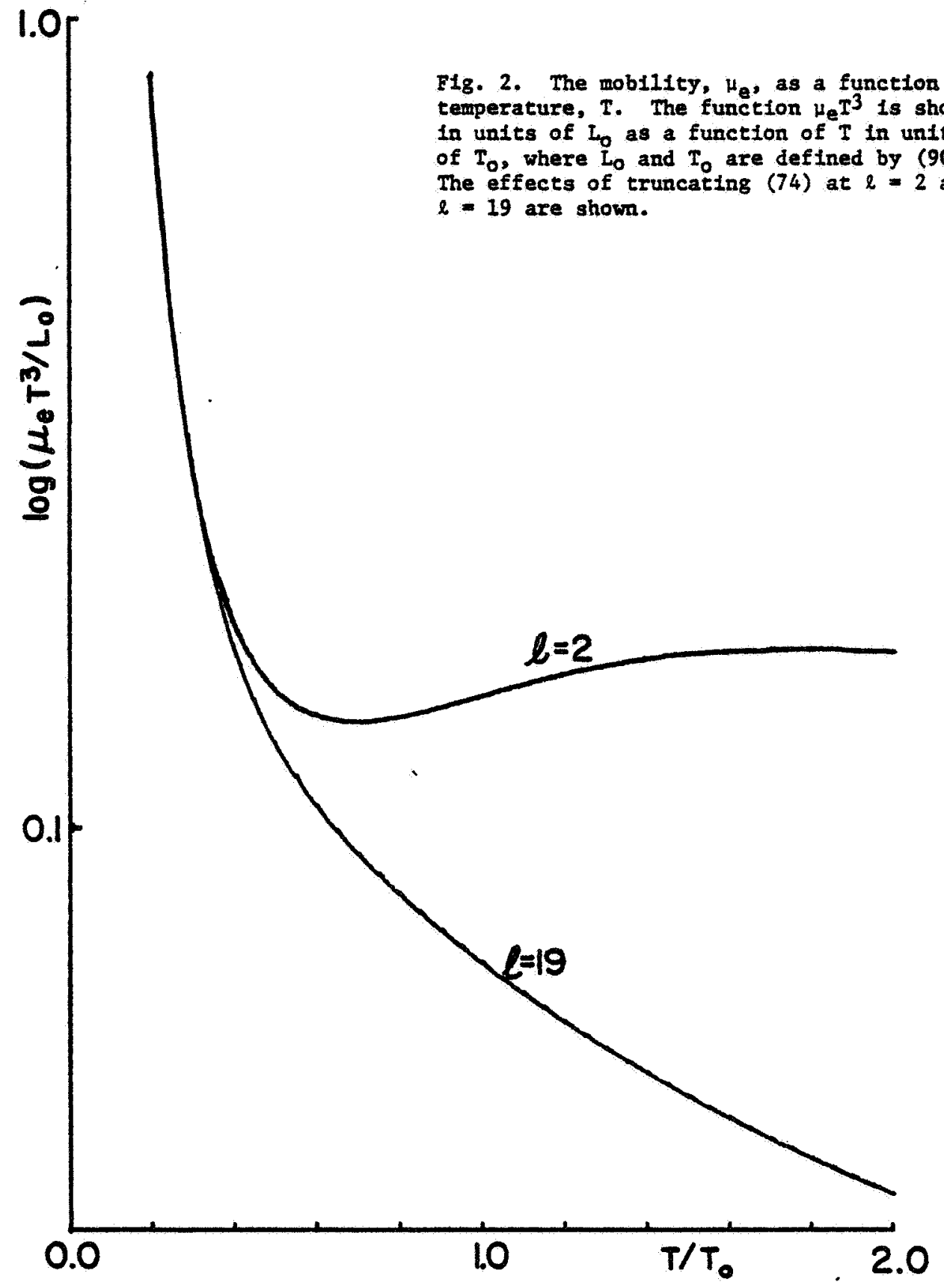

